\title{
Decentralized governance may lead to higher infection levels and sub-optimal releases of quarantines amid the COVID-19 pandemic
}

\author{
Adam Lampert ${ }^{1, *}$ \\ (1) Institute of Environmental Sciences, The Robert H. Smith Faculty of Agriculture, Food and \\ Environment, The Hebrew University of Jerusalem, Rehovot, Israel \\ * Corresponding author, Email: adam.lampert@mail.huji.ac.il
}

Feb. 11, 2022

\begin{abstract}
The outbreak of the novel Coronavirus (COVID-19) has led countries worldwide to administer quarantine policies. However, each country or state decides independently what mobility restrictions to administer within its borders, while aiming to maximize its own citizens' welfare.

4 Since individuals travel between countries and states, the policy in one country affects the infection levels in other countries. Therefore, major question is whether the policies dictated by multiple governments could be efficient. Here we focus on the decision regarding the timing of releasing quarantines, which were common during the first year of the pandemic. We consider a game-theoretical epidemiological model in which each government decides when to switch from a restrictive to a non-restrictive quarantine and vice versa. We show that, if travel between countries is frequent, then the policy dictated by multiple governments is sub-optimal. But if international travel is restricted, then the policy may become optimal.
\end{abstract}

Keywords: COVID-19, dynamic games, international policy, quarantine policy

Word count: abstract -147 words; main text -1498 words.

\section{Introduction}

The outbreak of the novel Coronavirus (COVID-19) necessitated quarantine policies, particularly during its early stages, before vaccines were available [1-4]. While mobility restriction measures have been taken during several disease outbreaks in history [5-11], the quarantines needed for the COVID-19 pandemic were at global scales that encompass entire countries. Such quarantines have economic and social costs $[12,13]$, and major relevant questions are how restrictive the quarantines should be, and what would be the right timing to release some of the mobility restrictions [1-3]. In practice, this decision is made independently by multiple countries [14-16], or independently by multiple states in countries like the U.S., or even independently by multiple municipal authorities [17]. Each governor might incline to dictate the strategy that best serves her/his own citizens; however, in periods when the quarantines are less restrictive, travelers can 
transmit the disease between countries, states, and cities. Consequently, the quarantine policy in one country/state may ultimately affect the outcome in other countries/states.

Such decentralized governance has a benefit: Each country or state may have a better knowledge of its own citizens' lifestyle and needs and may dictate a policy that better suits its own citizens. However, the decentralized policy also comes with a cost: Each government might ignore the cost borne to citizens of other countries due to international and interstate travel. Accordingly, various previous game theory studies have suggested that agents (individuals or countries) under-invest in the prevention and control of diseases [18-23].

In this paper, we examine the case where each governor decides independently about the timing of releasing the quarantine, and we ask what the inefficiency is due to such decentralized governance. Namely, we examine how the strategies of different governments differ from the socially optimal strategy of a hypothetical centralized government that aims to maximize the welfare of all the citizens in all the countries. Specifically, we consider two countries/states, and we analyze the following three cases (Fig. 1) [14-16, 24]: (1) the countries have approximately the same population size and infection level; (2) the countries have approximately the same population size, but one country experiences a more severe outbreak at a given point in time; and (Case 3 ) one state experiences a more severe outbreak compared to the rest of the country.

\section{Model}

Our model is general, but some of the assumptions and parameterization are motivated by the COVID-19 outbreak. We consider two states or countries, 1 and 2, and in line with data about early COPVID-19 outbreaks [4, 14, 16, 24-27], we assume that the number of infected people in each country is very small compared to the country's total population size. Accordingly, we consider only the dynamics of the infection level in each country, $I_{i}$, defined as the portion of individuals that are infected in country $i$. (Namely, in contrast to traditional SIR models [28], here we consider shorter timescales during which the number of susceptible individuals is constant, which is in line with the COVID-19 data from 2020, when quarantines were common [4, 14, 16, 24-27]).

We assume for simplicity that each government can administer one of two types of quarantine at any given time: restrictive and non-restrictive. Each government can choose when to switch between these two quarantines. We assume that under a restrictive quarantine in country $i, I_{i}$ decreases exponentially after the first two weeks at a rate $-r_{0}$ as long as $I_{i}>I_{\min }$. (Even a restrictive quarantine is not expected to eliminate the disease entirely, and $I_{\min }$ characterizes some minimal infection level that persists in the population.) In turn, according to evidence showing that the infection level may still increase under non-restrictive quarantine conditions [4, 
$r_{i}$ is country-specific and can be higher in those countries or states where interactions among individuals are more frequent. Also, we assume that if a country is under a restrictive quarantine, there is no travel from or to that country, whereas if both countries are under a non-restrictive quarantine, some individuals travel between these countries. We describe in more detail the dynamics of $I_{1}$ and $I_{2}$ in Methods: Dynamics of the infection levels.

In turn, the government in each country dictates the timing at which it switches from the nonrestrictive quarantine to the restrictive one and vice versa. We assume that a government will not allow the health system in its country to collapse [29], and therefore, it will always switch to a restrictive quarantine if $I_{i}$ approaches some maximum capacity, $I_{i}=I_{\max }$. In turn, under this constraint, the objective of each government is to maximize the proportion of time during which its own citizens are under a non-restrictive quarantine. We describe in more detail the objective functions in Methods: Objective functions.

Finally, we calculate the strategies of the governments in a Nash equilibrium and compare them to the socially optimal solution. In general, the Nash equilibria in such a dynamic game depend on various assumptions about the information that each government has [30]. Here we assume that each government does not get feedback about the infection level in the other country/state and decides in advance when it releases the quarantine. In our model, this results in a unique Nash equilibrium per a given set of parameter values (open-loop Nash equilibrium [30]). The numerical method that we used for finding the open-loop Nash equilibria and the optimal solutions is described in Methods: Numerical methods.

\section{Results \& Discussion}

Our results show that, in all three cases, if the number of travelers between the countries is greater than a certain threshold, the governments switch to a non-restrictive quarantine sooner compared to the socially optimal solution (Figs. 2,3). Namely, in Nash equilibrium, the governors administer the restrictive quarantine for shorter periods compared to the socially optimal solution. In turn, this results in shorter periods before the infection level approaches its full capacity and the restrictive quarantine is administered again (Figs. 2,3). Consequently, under decentralized governance, the solution is sub-optimal, the total amount of time during which a restrictive quarantine is administered is greater, and the average infection level is higher. This result is consistent with previous results that suggested that agents under-invest in the control of diseases [18-23]. Note that in both the optimal solution and the Nash equilibrium, the governments' actions tend to be synchronized as the second country to switch does so when its infection level is similar to that of the other country (Fig. 2D,F).

The difference between the optimal solution and the Nash equilibrium emerges if the number of 
medRxiv preprint doi: https://doi.org/10.1101/2020.05.20.20108167; this version posted February 11, 2022. The copyright holder for this preprint

(which was not certified by peer review) is the author/funder, who has granted medRxiv a license to display the preprint in perpetuity.

It is made available under a CC-BY-ND 4.0 International license .

of anarchy) increases with the number of travelers (Fig. 3). However, if the number of travelers is below the threshold, the Nash equilibrium and the optimal solution are identical (Fig. 3 ). This suggests that one way to prevent the inefficiency due to the decentralized governance is to restrict international or interstate travel to a low level even when the quarantines are nonrestrictive.

There are two mutually dependent reasons why decentralized governance results in releasing the restrictive quarantine sooner. First, each governor ignores the damages that its own travelers inflict on other countries, and thus, keeping a higher level of infection is perceived by the governor as less costly. Second, as a consequence of the first reason, each country hosts more infected travelers from the other country, and its own travelers are also hosted in countries with higher infection levels. Consequently, if the infection level in a given country is low, it increases rapidly due to travel, and therefore, it is not worthwhile for the country to reduce its infection level beyond a certain value (where due to travel, this value may be lower than $I_{\min }$ ).

Finally, note that we have made numerous simplifying assumptions in our model, which suggests various future directions for examining the consequences of relaxing these assumptions. First, we considered only two types of quarantine, whereas in reality, more options are available. In particular, governments can try to administer an intermediate level of quarantine that keeps the infection at a constant level. Examining whether this policy is better than the ones that we considered is beyond the scope of this paper (see $[2,3,29])$; however, a similar result will likely hold: Decentralized governance might maintain a higher infection level than the optimum. Second, we assumed that travel is allowed under non-restrictive quarantine. However, it would be interesting to examine policies that also dictate how to best integrate the quarantine policy with travel policy. Specifically, further restrictions on travel might mitigate the problem (Fig. 3); however, restrictions on travel come with economic costs [13]. Third, we considered only two countries, whereas considering more countries is generally expected to increase the price of anarchy [30]. And fourth, we considered open-loop solutions in which the governments predetermine their policy, but communication among the governments might lead to the formation of agreements and coordination of a more global quarantine policy.

\section{References}

1. Ferguson, N.M., et al., Impact of non-pharmaceutical interventions (NPIs) to reduce COVID-19 mortality and healthcare demand. DOI: https://doi.org/10.25561/77482, 2020.

2. Alvarez, F.E., D. Argente, and F. Lippi, A simple planning problem for COVID-19 lockdown. 2020, National Bureau of Economic Research.

3. Piguillem, F. and L. Shi, The optimal COVID-19 quarantine and testing policies. 2020, Einaudi Institute for Economics and Finance (EIEF). 
4. Fang, Y., Y. Nie, and M. Penny, Transmission dynamics of the COVID-19 outbreak and effectiveness of government interventions: A data-driven analysis. Journal of Medical Virology, 2020. 92(6): p. 645-659.

5. Ferguson, N.M., et al., Strategies for containing an emerging influenza pandemic in Southeast Asia. Nature, 2005. 437(7056): p. 209-214.

6. Glass, R.J., et al., Targeted social distancing designs for pandemic influenza. Emerging Infectious Diseases, 2006. 12(11): p. 1671.

7. Wallis, P. A dreadful heritage: Interpreting epidemic disease at Eyam, 1666-2000. in History Workshop Journal. 2006. Oxford University Press.

8. Halloran, M.E., et al., Modeling targeted layered containment of an influenza pandemic in the United States. Proceedings of the National Academy of Sciences, 2008. 105(12): p. 4639-4644.

9. Kohn, G.C., Encyclopedia of plague and pestilence: from ancient times to the present. 2007: Infobase Publishing.

10. Davis, R., The Spanish flu: narrative and cultural identity in Spain, 1918. 2013: Springer.

11. Tognotti, E., Lessons from the history of quarantine, from plague to influenza $A$. Emerging Infectious Diseases, 2013. 19(2): p. 254.

12. Atkeson, A., What will be the economic impact of COVID-19 in the US? Rough estimates of disease scenarios. 2020, National Bureau of Economic Research.

13. Eichenbaum, M.S., S. Rebelo, and M. Trabandt, The macroeconomics of epidemics. 2020, National Bureau of Economic Research.

14. Chinazzi, M., et al., The effect of travel restrictions on the spread of the 2019 novel coronavirus (COVID-19) outbreak. Science, 2020. 368(6489): p. 395-400.

15. Sohrabi, C., et al., World Health Organization declares global emergency: A review of the 2019 novel coronavirus (COVID-19). International Journal of Surgery, 2020.

16. Flaxman, S., et al., Report 13: Estimating the number of infections and the impact of nonpharmaceutical interventions on COVID-19 in 11 European countries. 2020.

17. Ren, X., Pandemic and lockdown: a territorial approach to COVID-19 in China, Italy and the United States. Eurasian Geography and Economics, 2020. 61(4-5): p. 423-434.

18. Francis, P.J., Dynamic epidemiology and the market for vaccinations. Journal of Public Economics, 1997. 63(3): p. 383-406.

19. Barrett, S., Global disease eradication. Journal of the European Economic Association, 2003. 1(23): p. 591-600.

20. Bauch, C.T. and D.J. Earn, Vaccination and the theory of games. Proceedings of the National Academy of Sciences, 2004. 101(36): p. 13391-13394.

21. Gersovitz, M. and J.S. Hammer, The economical control of infectious diseases. The Economic Journal, 2004. 114(492): p. 1-27.

22. Barrett, S., The smallpox eradication game. Public Choice, 2007. 130(1-2): p. 179-207.

23. Fenichel, E.P., Economic considerations for social distancing and behavioral based policies during an epidemic. Journal of health economics, 2013. 32(2): p. 440-451.

24. World-Health-Organization, Coronavirus disease 2019 (COVID-19): situation report, 72. 2020.

25. Linton, N.M., et al., Incubation period and other epidemiological characteristics of 2019 novel coronavirus infections with right truncation: a statistical analysis of publicly available case data. Journal of Clinical Medicine, 2020. 9(2): p. 538.

26. European Centre for Disease Prevention and Control. 2020; https://www.ecdc.europa.eu/en/geographical-distribution-2019-ncov-cases].

27. COVID-19 Data Repository by the Center for Systems Science and Engineering (CSSE) at Johns Hopkins

University.

2020; 


\section{https://www.arcgis.com/apps/opsdashboard/index.html\#/bda7594740fd40299423467b48e9ecf}

6].

28. Anderson, R.M. and R.M. May, Population biology of infectious diseases: Part I. Nature, 1979. 280(5721): p. 361-367.

29. Ferguson, N., et al., Report 9: Impact of non-pharmaceutical interventions (NPIs) to reduce COVID19 mortality and healthcare demand. 2020.

30. Dockner, E.J., et al., Differential games in economics and management science. 2000: Cambridge University Press.

31. Bichara, D., et al., SIS and SIR epidemic models under virtual dispersal. Bulletin of Mathematical Biology, 2015. 77(11): p. 2004-2034.

32. Castillo-Chavez, C., D. Bichara, and B.R. Morin, Perspectives on the role of mobility, behavior, and time scales in the spread of diseases. Proceedings of the National Academy of Sciences, 2016. 113(51): p. 14582-14588.

33. Clark, C., Mathematical bioeconomics: The Mathematics of Conservation. Third ed. 2010: Joan Wiley \& Sons. 

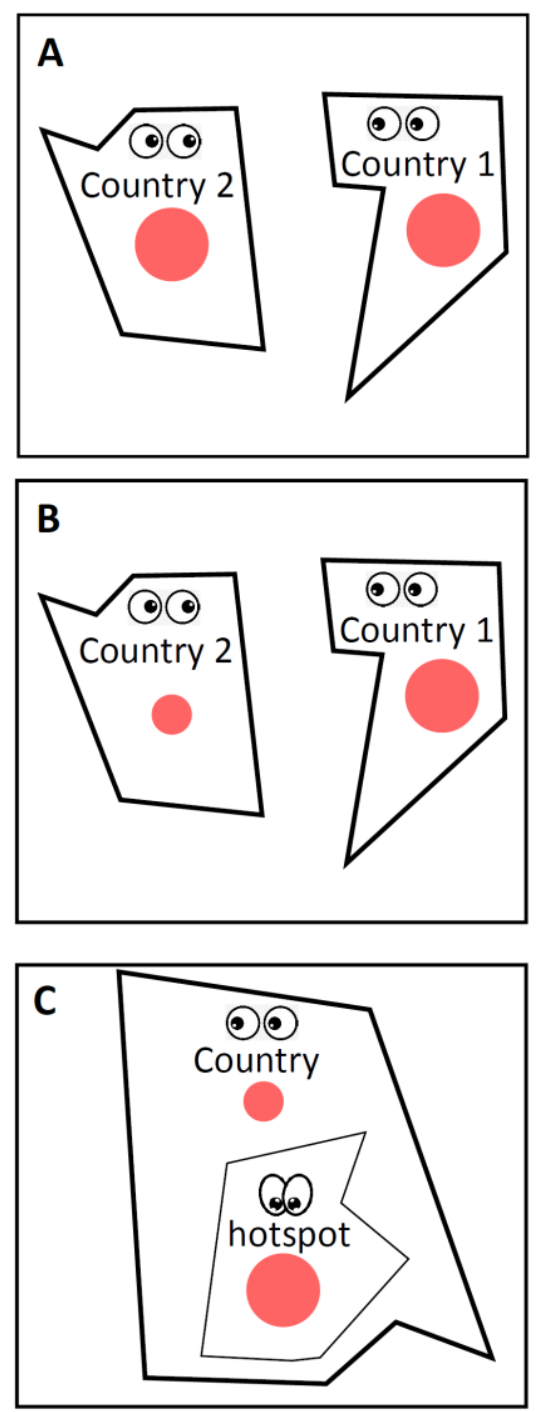

118 Figure 1: Illustration of the three cases that we consider. (A) Case 1: Both countries have the 119 same population size and infection level. (B) Case 2: Both countries have the same population 120 size, but country 1 has a more severe outbreak. (C) Case 3: One U.S. state is a "hotspot" and has 121 a more severe outbreak than the rest of the U.S. 

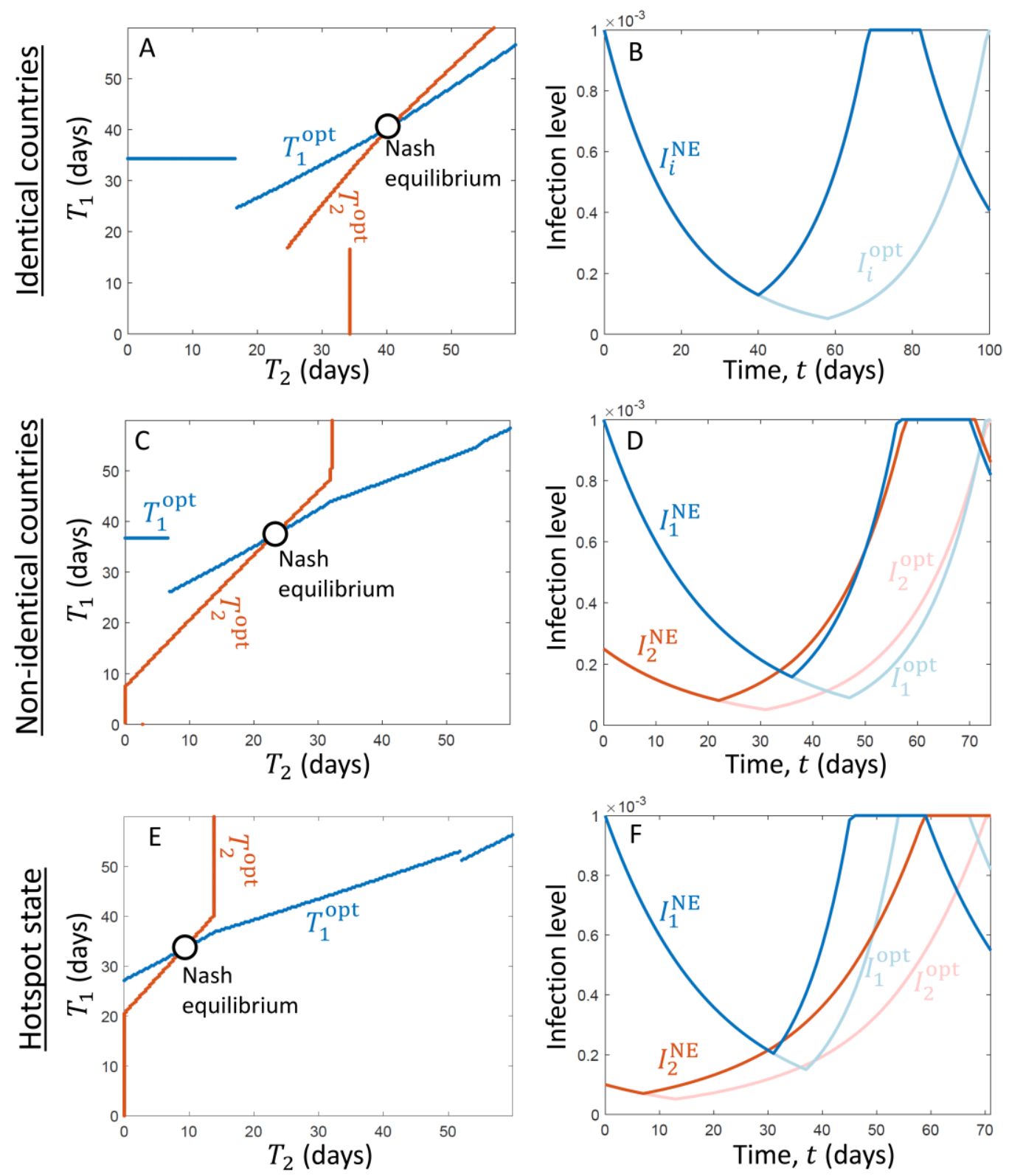

Figure 2: The equilibrium solution dictates that governments release the quarantines earlier than optimal. We consider the three cases illustrated in Fig. 1: identical countries (A, B), nonidentical countries $(C, D)$, and a state that initially has a much higher infection level than the rest of the U.S. $(E, F)$. The left column (A, C, E) shows the optimal time for country 1 to release the quarantine, $T_{1}^{\mathrm{opt}}$, as a function of the day when country 2 releases the quarantine, $T_{2}$ (blue line). It also shows the optimal time for country 2 to release the quarantine, $T_{2}^{\text {opt }}$, as a function of the day when country 1 releases the quarantine, $T_{1}$, on a flipped axis (mirror image, red line). The intersection of the blue and the red line indicates the open-loop Nash equilibrium. In turn, the right column (B, D, E) shows the time evolution of the infection level in countries 1 and 2, assuming that they adopt the Nash equilibrium strategies $\left(I_{1}^{\mathrm{NE}}\right.$, blue line, and $I_{2}^{\mathrm{NE}}$, red line), as 
132 well as the infection levels assuming that the countries adopt the socially optimal solution $\left(I_{1}^{\text {opt }}\right.$, 133 light blue line, and $I_{2}^{\text {opt }}$, light red line). In all three cases, the governments release the quarantine 134 sooner than the optimum if they follow the Nash equilibrium. Moreover, in both the optimal 135 solution and the Nash equilibrium, the second country to release the quarantine does so 136 approximately when its infection level approaches that of the other country.

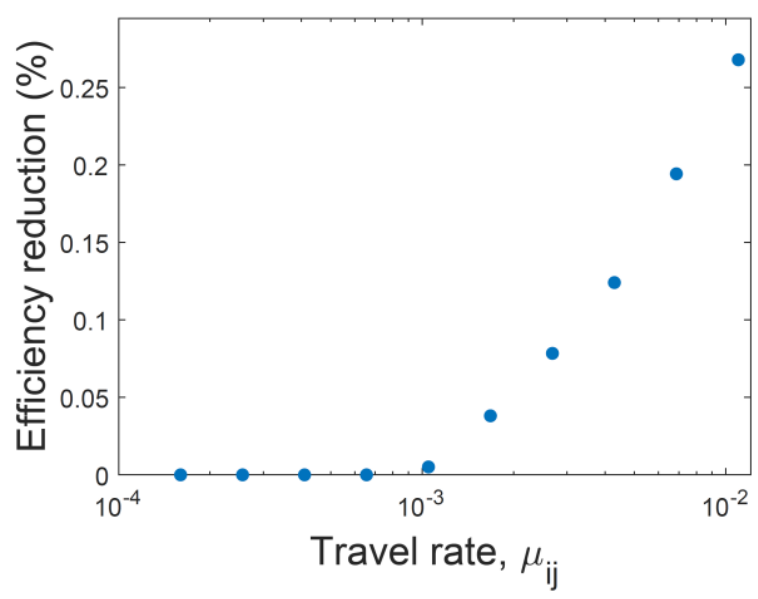

137 Figure 3: Travel between states may lead to inefficient quarantine policy within each state. 138 Demonstrated is the increase in the relative time that a restrictive quarantine is administered 139 (efficiency reduction, a.k.a. "cost of anarchy") in a Nash equilibrium (decentralized government) 140 compared to the optimal solution. The efficiency reduction is zero if the proportion of travelers 141 in the population (travel rate, $\mu_{i j}$ ) is below a certain threshold, and increases with $\mu_{i j}$ above that 142 threshold. 


\section{Supplementary Online Materials}

\section{Methods}

\section{Dynamics of the infection levels}

143 Here we describe in detail the dynamics of $I_{1}(t)$ and $I_{2}(t)$, which characterize the proportions of 144 infected individuals in the populations of countries 1 and 2, respectively. Assume that each of the two countries can be under a restrictive quarantine or a non-restrictive quarantine, and the type of quarantine can change over time. The dynamics of $I_{i}$ at time $t$ depend on the type of quarantine administered in both countries at that time.

Option 1: Country $i$ is under a restrictive quarantine.

149 If country $i$ is under a restrictive quarantine at time $t, I_{i}(t)$ declines at a daily rate $-r_{0}$, regardless 150 of what happens in the other country (no travel). However, there are two exceptions. First, $I_{i}$ 151 cannot decline below a certain threshold, $I_{\min }$, characterizing the minimal level of infection in 152 the population. Second, if the government switches from restrictive to non-restrictive 153 quarantine, $I_{i}$ starts to decline only after a delay of $T_{\text {delay }}$ days. Therefore, in summary,

$$
\frac{d I_{i}}{d t}=\left\{\begin{array}{ccc}
r_{0} I_{i} & \text { if } I_{i}>I_{\min } \text { and } t_{s}>T_{\text {delay }} \\
0 & \text { otherwise }
\end{array}\right.
$$

where $t_{s}$ is the time (in days) from the day when the quarantine became restrictive.

Option 2: Country $i$ is under a non-restrictive quarantine, but the other country, $j \neq i$, is under a restrictive quarantine.

157 In this case, there is still no travel between the countries due to the restrictive quarantine in 158 country $j$. Consequently, $I_{i}$ grows exponentially at a daily rate $r_{i}$ :

$$
\frac{d I_{i}}{d t}=r_{i} I_{i}
$$

Option 3: Both countries are under a non-restrictive quarantine

160 In this case, individuals travel between the countries, where $\mu_{i j}$ of the residents of country $i$ are 161 in travel to country $j$. If we assume that the number of travelers from a country is much smaller 162 than the number of the country's own residents $\left(\mu_{i j} \ll N_{i}\right.$ and $\left.\mu_{j i} \ll N_{j}\right)$, it follows that

$$
\frac{d I_{i}}{d t}=r_{i} I_{i}+\frac{r_{i} \mu_{j i}+r_{j} \mu_{i j}}{N_{i}} I_{j}
$$


Specifically, (i) the term $r_{i} I_{i}$ characterizes residents of country $i$ that get infected inside country $i$ by other residents of country $i$; (ii) the term $r_{i} \mu_{j i} / N_{i}$ characterizes residents of country $i$ that get infected inside country $i$ by residents of country $j$; and (iii) the term $r_{j} \mu_{i j} / N_{i}$ characterizes residents of country $i$ that get infected inside country $j$ by residents of country $j$. Note that we assume that travel is temporary (Lagrangian approach [31, 32]); that is, a traveler from country 1 to country 2 is still a resident of country 1 who will return to country 1 , and therefore, is s/he gets infected, it increases $I_{1}$, even the infection has occurred in country 2.

In turn, without the simplifying assumption that $\mu_{i j} \ll N_{i}$, Eq. (3) can be written in a more general form:

$$
\frac{d I_{i}}{d t}=\left[\frac{r_{i}\left(N_{i}-\mu_{i j}\right)^{2}}{N_{i}-\mu_{i j}+\mu_{j i}}+\frac{r_{j} \mu_{i j}^{2}}{N_{j}-\mu_{j i}+\mu_{i j}}\right] \frac{1}{N_{i}} I_{i}+\left[\frac{r_{i}\left(N_{i}-\mu_{i j}\right) \mu_{j}}{N_{i}-\mu_{i j}+\mu_{j i}}+\frac{r_{j} \mu_{i j}\left(N_{j}-\mu_{j i}\right)}{N_{j}-\mu_{j i}+\mu_{i j}}\right] \frac{1}{N_{i}} I_{j} .
$$

In our simulations, we used values of $\mu_{i j}$ that are much smaller than $N_{i}$ (see Parameterization subsection), and therefore, the results obtained using Eq. (3) and those obtained using Eq. (4) were almost indistinguishable.

\section{Objective functions}

The government in each country chooses the time periods during which each type of quarantine is administered in that country. We assume that the objective of each government is to maximize the relative time during which the quarantine is non-restrictive in its country, under the constraint that the health system does not collapse, and therefore, a restrictive quarantine has to be administered in country $i$ whenever $I_{i}$ approaches $I_{\max }$ [29].

Specifically, denote $T_{i}^{\text {tot }}$ as the time to complete a cycle during which $I_{i}$ increases to $I_{\max }$ and returns back to its initial value, $I_{i}(0)$. In turn, denote $T_{i}^{\text {non }}$ as the total time within such a cycle during which a non-restrictive quarantine is administered in country $i$. Then, we define the utility of country $i$ as

$$
u_{i}=\frac{T_{i}^{\text {non }}}{T_{i}^{\text {tot }}}
$$

In turn, we calculate both the optimal solution and the open-loop Nash equilibrium. Specifically, the optimal strategy is the one that maximizes $N_{1} u_{1}+N_{2} u_{2}$. The open-loop Nash equilibrium is given by the set of pre-determined strategies that are such that no country can increase its own utility by unilaterally changing its strategy.

\section{Numerical methods}


Since the growth rates of $I_{1}$ and $I_{2}$ are piecewise-linear, and since we considered a time delay after the government switches to restrictive quarantine before the infection level starts to decline, it follows from Pontryagin's maximum principle $[30,33]$ that the optimal strategy of each government is to choose a time, $T_{i} \geq 0$, at which it switches to a restrictive quarantine and then to wait until $I_{i}=I_{\max }$ before switching to a restrictive quarantine again (Fig. 2).

Then, $T_{i}^{\text {non }}$, is the period from $T_{i}$ until $I_{i}$ approaches $I_{\max }$, and the time during which the quarantine is restrictive, $T_{i}^{\text {tot }}-T_{i}^{\text {non }}$, is given by the sum of three distinct periods: (i) between $t=0$ and $t=T_{i}$, (ii) $T_{\text {delay }}$ days after $I_{i}$ approaches $I_{\max }$, and (iii) the time until $I_{i}$ declines back to its initial value, $I_{i}(0)$.

To find the optimal solution and the open-loop Nash equilibrium, we first generate a matrix in which each cell characterizes a set of strategies, $\left(T_{1}, T_{2}\right)$, and we simulate dynamics to calculate the utility for each country in every cell. Then, the optimal solution is given by $T_{1}$ and $T_{2}$ that correspond to the cell in which $N_{1} u_{1}+N_{2} u_{2}$ is maximized. In turn, the open-loop Nash equilibrium is found by calculating the best response of each country to every strategy of the other country, where the intersection of the curves characterizes the Nash equilibrium (Fig. 2 A,C,E). The values of $T_{1}$ and $T_{2}$ in the matrix vary between 0 and $T_{\max }$, where $T_{\max }$ is the time when a restrictive quarantine reduces the infection level in both countries to $I_{\mathrm{min}}$. (It is never worthwhile to keep a restrictive quarantine for more than $T_{\max }$ days.) In turn, the size of the matrix determines the resolution at which the strategies $T_{1}$ are $T_{2}$ are examined, and in Fig. 2, we used a $200 \times 200$ matrix.

\section{Parameterization}

The estimations of the parameter values used for the simulation are taken from datasets and literature related to outbreaks of COVID-19 during quarantines [4, 14, 16, 24-27]. Notice that some parameter values vary significantly between countries and states, and therefore, we performed sensitivity analyses to verify that the main results are general and hold within wider parameter ranges.

Under restrictive quarantine conditions, a daily decline rate of $5 \%$ in the infection level after the first two weeks is a reasonable estimate. For example, in China, the number of infected individuals declined from $\sim 86,000$ to $\sim 3,000$ within 76 days, which implies $r_{0} \approx-5 \%$ per day. In turn, in several countries, it took about 14-21 days before any decline occurred following a quarantine, which suggests that considering $T_{\text {delay }} \approx 14$ days is reasonable. Next, note that $r_{i}$ depends on the restrictions used in a given non-restrictive quarantine, and it may vary between states and countries. We used estimates that reflect the weeks before a restrictive quarantine was administered in countries in Europe, where $5 \% \leq r_{i} \leq 15 \%$ per day is a reasonable estimate. 
222 In turn, we assume that $I_{\max }$ is given approximately by the infection level that was approached

223 in countries in Europe before the restrictive quarantine was administered or two weeks after it

224 was administered, where considering $I_{\min } \approx 0.01 \%-0.1 \%$ of the total population size is a

225 reasonable estimate. Next, parameters like $I_{\min }$ and the travel rates $\mu_{12}$ and $\mu_{21}$ are harder to

226 estimate, as they depend on the specific location and scale of the countries and states

227 considered. Therefore, we performed sensitivity analyses and examined a variety of values (e.g.,

228 Fig. 3). Finally, the ratio between $N_{1}$ and $N_{2}$ reflects the relative population sizes of the two 229 countries: $N_{1}=N_{2}$ characterizes the cases demonstrated in Fig. 1A,B and 2A-D, while $N_{1}<N_{2}$ 230 characterizes the case demonstrated in Fig. 1C and 2E-F.

\section{Parameter values used to generate the figures}

231 Fig. $2 \mathrm{~A}, \mathrm{~B}: r_{0}=-5 \%\left(\mathrm{day}^{-1}\right), r_{1}=r_{2}=7 \%\left(\mathrm{day}^{-1}\right), I_{\max }=0.1 \%, I_{\min }=0.005 \%, T_{\text {delay }}=$

23214 (days), $N_{1}=N_{2}=1, \mu_{12}=\mu_{21}=0.2 \% \times N_{1}$.

233 Fig. 2C,D: $r_{0}=-5 \%\left(\right.$ day $\left.^{-1}\right), \quad r_{1}=9 \%\left(\right.$ day $\left.^{-1}\right), \quad r_{2}=7 \%\left(\right.$ day $\left.^{-1}\right), I_{\max }=0.1 \%, \quad I_{\min }=$ $2340.005 \%, T_{\text {delay }}=14$ (days), $N_{1}=N_{2}=1, \mu_{12}=\mu_{21}=0.25 \% \times N_{1}$.

235 Fig. 2E,F: $r_{0}=-5 \%\left(\right.$ day $\left.^{-1}\right), \quad r_{1}=11 \%\left(\right.$ day $\left.^{-1}\right), \quad r_{2}=5 \%\left(\right.$ day $\left.^{-1}\right), \quad I_{\max }=0.1 \%, \quad I_{\min }=$ $2360.005 \%, T_{\text {delay }}=14$ (days), $N_{1}=0.3, N_{2}=1, \mu_{12}=0.25 \% \times N_{1}, \mu_{21}=0.25 \% \times N_{2}$.

237 Fig. 3: $\mu_{12}=\mu_{21}$ varies (x-axis), and the other parameters are the same as those in Fig. $2 \mathrm{~A}, \mathrm{~B}$. 